\title{
Resonant elastic X-ray scattering: Where from? where to?
}

\author{
C. Vettier ${ }^{\mathrm{a}}$
}

European Synchrotron Radiation Facility, 38043 Grenoble, France

Received 9 January 2012 / Received in final form 23 March 2012

Published online 15 June 2012

\begin{abstract}
The International Conference on Anomalous Scattering held in Malente, Germany, in 1992 examined the broad issue of 'dispersion or resonant scattering' for X-rays. The significant aspects discussed at that time included: the evaluation of X-ray scattering factors, the role of local broken symmetries and the application to structure determinations, the impact on magnetic X-ray scattering, but also resonant Raman scattering and nuclear resonant scattering. However, many aspects of resonant elastic X-ray scattering (REXS) were still being revealed and soon it was realised that new productive paths of research would open up. In the last two decades, applications have developed thanks to concurrent developments in X-ray instrumentation and theoretical approaches. Focusing on REXS only, noteworthy fields are: the observation of multiple order parameters, the detection of multipolar moments, but also applications in chemistry and materials sciences, macromolecules and soft matter in the soft X-ray range. REXS has evolved from the exploitation of large resonant signals to a powerful experimental and characterisation method aimed at unravelling new phenomena. The recent technical and theoretical advances have paved the way to further REXS developments in many fields of science.
\end{abstract}

\section{Introduction}

One of the many benefits of synchrotron radiation is the ease of energy adjustment of the X-ray beams that allows detailed investigations of scattering processes near absorption edges and the exploitation of the resonant process. The contributions given at the Malente Conference [1] in 1992 demonstrated clearly that the advent of synchrotron sources had led to major advances in the field. As the late David Templeton put it [2], the previous Inter-Congress Conference on Anomalous Scattering held in Madrid in 1974 had marked "the end of the X-ray tube and the beginning of the storage ring as the source of choice for these experiments". Until synchrotron radiation could be used, large efforts had been put on the estimation of resonant of the X-ray scattering factors $[3,4]$ while the impact of X-ray anomalous and resonant scattering on structures determination had been very small.

${ }^{a}$ e-mail: vettier@esrf.eu 
The Malente meeting revealed that resonant X-scattering was on the verge of expanding in many directions thanks to the synchrotron sources of the so-called second generation. At that time, the meeting illustrated the experimental results obtained at second-generation synchrotron sources and theoretical advances. The major points that were presented involved magnetic X-ray scattering, the applications of resonant scattering to structure determinations, Resonant Inelastic X-ray Scattering, the role of anisotropic scattering factors, and the determination of optical constants and atomic scattering factors. Furthermore, stimulated by experimental results [5-8] the theoretical understanding of the resonant process had improved [9], and deeper analysis of experimental results became possible [10].

Malente marked the advent of third generation synchrotron sources with highly tuneable X-ray beams, good energy resolution and efficient instrumentation; these developments were more or less paralleled by advances on the theoretical front, including realistic modelling of resonant scattering amplitudes. Since then, the growing availability of synchrotron X-ray sources, but also the impressive progress in instrumentation and theoretical modelling have prompted an enormous growth of resonant X-ray scattering methods. Resonant X-ray Scattering has evolved from being a very exciting and promising field of research and/or a very efficient intensity enhancer to being an extraordinary rich probe of the various states of matter. This has been quite visible in some areas such as local symmetry properties in solids, materials sciences, soft matter and electronic properties in condensed matter.

Since the Malente conference, so many applications of Resonant X-ray Scattering have burgeoned that the organisers of the present conference decided to focus on Resonant Elastic X-ray Scattering (REXS), an elastic phonon-in phonon-out process. It should be noted that, at least two other fields have led to promising developments: Resonant Inelastic X-ray Scattering [11], in particular in the field of magnetic excitations [12], and the various X-ray dichroism methods [13].

More progress is still to be expected: nano-focusing of X-ray beams, enhanced energy resolution, and the availability of coherent X-ray beams that could allow fast time-resolved studies will open new avenues for research on the electronic and structural properties of matter.

\section{Resonant elastic scattering: A unique probe}

REXS is about the detection, the characterisation and the understanding of scattered X-rays intensities from sample materials when the X-ray energy is tuned near absorption edges of the material components. Several approaches to REXS have been put forward $[9,10,14-17]$. Briefly speaking, REXS originates from virtual multipole transitions induced by the incident radiation near absorption edges; as such REXS is very sensitive to the local environment and bonding configuration. REXS exhibits unique features:

- REXS is element specific. The chemical sensitivity arises from the element specificity of absorption edges.

- REXS is orbital specific. The choice of the absorption edges selects the electronic core level to be excited, and different final states can be probed through multipole transitions.

- REXS is a sensitive probe of multipolar moments. Applications of symmetry arguments can distinguish magnetic and non-magnetic effects and predict how optical activity can be observed $[9,10,13,18-20]$.

- REXS intensities have a dependence on the photon energy that contains rare information on energy scales in the system. 
Because of its extreme sensitivity to symmetry, REXS has the following characteristics:

- REXS exploits the polarisation of the photon beams. Both linear and circular polarisations of X-ray beams are to be considered [21].

- REXS depends on the scattering geometry. REXS probes the symmetry of local fields; varying the orientation of these fields with respect to the scattering plane (azimuthal scans) is a further probe of the origin of REXS [22].

There have been many improvements in the theoretical modelling of the resonant process. The resonant scattering amplitudes are related to matrix elements of multipole moments operators between the initial state and intermediate unoccupied electronic states. Classifications of the transition matrix elements have been achieved [18-20] based on their symmetry properties under space-inversion, rotation and time reversal. Realistic calculations of the energy dependence near the absorption edges can now be obtained through efficient computer packages and ab-initio electronic band calculations [23]. In the special case of resonant X-ray spectroscopy, extremely useful sum rules have also been derived that relate ground state properties (averaged values of moments) to integrals of resonant signals over photon energy [24]; similar derivations were proposed in the case of REXS [25]. The large amount of interest in resonant scattering methods at the time of the Malente Conference has initiated significant theoretical work, which in turn has allowed the surge of experimental work when experimental environment became available.

\section{Synchrotron X-ray instrumentation}

Indeed, dramatic progress in X-ray instrumentation has taken place during the last two decades. The most valuable aspect has been the development of new tools to tailor $\mathrm{X}$-ray beams: choice of the photon energy, beam stability, energy resolution, control of the X-ray polarisation. We shall consider the properties of X-ray sources, the characteristics of X-ray beams, properties at sample position and instrumentation/sample environment.

Obviously, the performance of modern storage rings [26] and the development of new insertion devices [27] have facilitated experiments involving the tuning of incident photon energy. Nowadays, effective instrument control allows scanning the photon energy while adjusting X-ray-source flux at its maximum and maintaining the scattering geometry. The relative incident energy resolution which is usually available on most instruments is in the $10^{-5}$ to $10^{-4}$ range. Although this represents quite an achievement, some progress might still be made in the future without reducing excessively the photon flux at the sample position.

The control of X-ray beam polarisation is an essential ingredient to REXS experiments. Helical undulators have been developed recently [28] that can produce any kind of polarisation of the incident X-ray beams, especially in the soft X-ray range. However, the transfer of the polarisation of the X-ray beam to the sample through the monochromator remains an issue. In parallel, X-ray phase plates have been designed to obtain circular polarisation from linearly polarised beams, using the perfect crystal birefringence near the Bragg diffraction condition as predicted by the X-ray dynamical theory [29]. Perfect diamond crystals and mosaic crystals such as beryllium can produce high degree of circular polarisation with a wide range of energy tenability. Furthermore, phase plates can be used to rotate linear polarisation in any direction perpendicular to the incident X-ray beam $[22,30]$. Such a set-up permits scans of the incident polarisation; combined with linear polarisation analysis of the X-ray scattered beam, this method allows full polarisation analysis. In vacuum linear polarisation- (and energy-) analysers are now in use at several synchrotron facilities. 
Better X-ray optics and brighter sources can produce highly focused X-ray beams (below $100 \mathrm{~nm}$ ) [31]. These highly focused X-ray beams available nowadays allow scanning probe techniques that are used in combination with REXS to probe structural and magnetic properties of materials. Furthermore, partial coherence of X-ray beams can now be achieved at many facilities, and can be exploited to investigate configurations in materials (composition and stress distributions, growth of nano-objects, ...) $[32]$.

Instruments and instrument control software have become so flexible and reliable that 'exotic' scans can be thought of without resorting to continuous human intervention. Since the days of Malente, most publications dealing with REXS on single crystal involve extensive X-ray polarisation analysis and/or azimuthal scans of the sample. Similarly, sample environment has developed (temperature, applied external fields, ... ) up to a point that the main limitations are not with experimental capabilities any more. There is however a blocking point with X-ray experiments at very low temperatures; it appears that bringing the scattering volume of the sample below $1 \mathrm{~K}$ remains a difficult task because of the heat load deposited by the synchrotron beams.

The generic features of REXS can now be fully exploited at all modern synchrotron sources where new instrumentation has been implemented. In the following, I shall give a few examples of experimental studies that illustrate the evolution of REXS during the last two decades.

\section{Deciphering local site symmetry properties}

One of the highlights at the Malente conference was the evidence of forbidden reflections observed in hematite by K.D. Finkelstein [8]. Forbidden Bragg reflections had been observed already before in particular by D.H. Templeton and L.K. Templeton [6], but this work on hematite [8] marked the start of renewed interest in that field. Anisotropic crystalline environments lead distortions of the electronic wave functions, and therefore the atomic scattering factors cannot be considered as scalar quantities any longer and should be treated as tensors. In some space groups, where resonant atoms occupy sites with different orientations (presence of screw-axis and/or glide plane), forbidden reflections can occur near absorption edges [7]. Experimental observations have included many ingredients of today's activities in REXS, namely energy lineshape of intensities, X-ray polarisation dependence and variation with azimuthal angle [33]. Analysis of the forbidden reflections has started with the modelling of symmetry adapted anisotropic tensors representing the anisotropic susceptibility. Originally all effects were considered to arise from dipole contributions only, but rather quickly, it became obvious that quadrupole contributions [8] and also mixed dipole-quadrupole terms had to be taken into account [34]. However, the various contributions to forbidden reflections near absorption edges can become very much entangled, especially if several order parameters, such as magnetic or orbital order, are involved (see below). In order to understand fully the various contributions and to account for the experimental data, extremely careful studies of the polarisation dependence, the azimuthal angle dependence over a very large angular span must be undertaken.

One such example is a recent work on hematite by Kokubun et al. [33] which has shown that mixed dipole-quadrupole and quadrupole-quadrupole scatterings together with non-resonant magnetic scattering (due to magnetic order in hematite) can explain all observations. It appears that REXS in these materials can become an exquisite probe for ferromagnetic S-domains because of the coupling between nonresonant magnetic scattering and resonant electric scattering. As a consequence, it 
can be envisaged to study the Dzyaloshinskii-Moriya interaction that leads to weak ferromagnetism in systems such as hematite [35].

Similar studies can also be performed in the soft X-ray range, where only a few Bragg peaks can be observed. One example is provided by the work of Tanaka et al. [36] who have shown that the use of circular polarisation can reveal handedness of crystals. More generally, such methods could be applied to a wide range of chiral motifs such as liquid crystals [37] or ferroelectrics and multiferroics [38].

In some high symmetry systems, such as diamond structures, the forbidden Bragg reflections that violate glide-plane or screw-axis selection rules can also reveal information about the dynamics of atoms, as shown in the case of Germanium [39]. It was observed that forbidden reflection intensities in Ge vary strongly with temperature, as it is expected if the anisotropy of the scattering factors originates from Ge atoms motions. Indeed, careful modelling [40] has evidenced that the thermal-motion-induced anisotropy is proportional to atomic displacements. It should be noted that resonant $\mathrm{X}$-ray diffraction experiments are performed with an energy resolution in the order of $\mathrm{eV}$; such an energy scale is not fully suited to lattice dynamics; improving the incident energy resolution down to $10-50 \mathrm{meV}$ would certainly allow more detailed investigations.

Although this conference focuses on elastic photon-in/photon-out experiments, it is worth mentioning the fascinating developments in the analysis of the various forms of X-ray dichroism [41]. The mixing of contributions from different multipolar moments with opposite parities can lead to a full scale zoology of optical activities, even in the X-ray range [42]. In connection to these experiments that detect the imaginary part of scattering factors in the forward geometry, it is tempting to relate the observed signals to observable quantities, the expectation values of related operators in the electronic ground state of the system. Sum rule formalisms have been developed over the years $[10,19,43,44]$.

\section{Characterising materials}

The availability of instruments for materials sciences at many synchrotron sources has led to an escalating interest in the exploitation of resonant scattering for structural studies. The importance of 'anomalous' scattering was first noted by J.M. Bijvoet [45]. There have been significant developments in the field of powder diffraction [46], but also in the field of structure determinations of macromolecular assemblies $[47,48]$ with the multi-wavelength anomalous diffraction (MAD); this last point will be further discussed in the next paragraph and in the paper by the late Richard Kahn [49]. The MAD phasing methods have revolutionised the field of macromolecular crystallography. As predicted 20 years ago [50], the MAD techniques have now become a routine tool to solve the 'phase problem' in order to reveal the molecular arrangement in a large variety of proteins of great biological interest $[51,52]$. Since the early days, MAD methods have matured as a routine tool and can deliver rapid solutions for biological structures [49]. Once again, this has been made possible through the combination of progress in instrumentation [52], in particular automation, and software development.

The MAD methods have also been used in materials sciences studies. As examples, it has been possible by observing the variations of intensities near absorption edges, to extract information on atomic displacements in incommensurately ordered crystals $[53,54]$ or even in magnetic superlattice systems [55] but also to detect changes in small inorganic molecules [56].

Following the increased performances of synchrotron sources, there has been a rapidly growing attraction for resonant diffraction experiments [57], including 
resonant contrast diffraction, diffraction anomalous fine structure (DAFS), anomalous small-angle X-ray scattering (ASAXS), grazing incidence diffraction combined with MAD methods or even grazing incidence small angle scattering near resonances $(\mathrm{A}+\mathrm{GISAXS})$. These methods have progressed owing to developments in instrumentation (X-ray optics, detectors) but also to the progress in specific schemes for data collection and on-line data analysis [58].

DAFS $[59,60]$ combines diffraction and X-ray absorption fine structure in order to provide long-range structural information together with site-sensitive and chemically selective information. DAFS methods have evolved towards applications dedicated to materials sciences, such as element selective observations of interfaces [61]. Similarly, it has been shown that DAFS spectroscopy combined with MAD can be exploited to obtain structural information of nanostructures, in particular nano-islands or nanowires grown on $\mathrm{Si}$ (001) [62]. These methods combined with MAD have made it possible to fully analyse strain and composition of nanostructures and the growth of nanostructures that play a crucial role in new functional materials [63].

In grazing incidence geometry, REXS experiments can provide direct determinations of strain and/or composition profiles in self-assembled structures [64,65] to complement microscopy techniques. Other applications concern the conformations of nano-particle networks either in three-dimensional forms or aggregated on surfaces. A+GISAXS [66] and ASAXS [67] studies have been shown to be pertinent methods to investigate nanostructures of metal/organic hybrids.

The obvious advantage of resonant scattering is to provide intensity contrast which allows for the possibility to distinguish neighbouring chemical elements in the periodic table. It is very similar to the isotope labelling methods used in neutron scattering techniques. However, the resonant X-ray methods have the unique advantage that they can be exploited with a unique sample by tuning the photon energy next to the various absorption edges of interest. The chemical sensitivity of resonant $\mathrm{X}$-ray diffraction allows the extraction of the contributions of the 'resonant' element in a given crystallographic site to the diffraction pattern. Fields of application of resonant contrast diffraction have expanded in the last decades [68] to cover powders with mixed-occupancy sites, or multi-phase studies, environmental studies to localise heavy atoms, extraction of partial structure factors in disordered materials [69] and even the determination of valence states through by the use of diffraction anomalous near-edge structure spectroscopy [70].

\section{Coping with soft matter}

Soft matter systems, including biological systems, represent a special case, because the usual components do not show resonant edges at X-ray energies that are suitable for wide angle diffraction experiments.

In the case of polymers with high $\mathrm{Z}$ counterions or in the case of metalloproteins, high $\mathrm{Z}$ edges can be exploited in the hard X-ray range. If no suitable high $\mathrm{Z}$ element is present in the macromolecules, extrinsic labelling techniques are used either to insert heavy atoms such as Se, Sr or lanthanides, or to substitute light metal atoms with heavier ones [49,71-73].

Nevertheless, recent developments in instrumentation have led to the development of beam lines dedicated to soft X-ray resonant scattering experiments, essentially SAXS [74] an reflectometry [75,76].

In the field of polymer solutions, microcapsules, and membranes or composite materials, which are essentially composed of low Z elements, soft X-ray resonant scattering has become a powerful complementary tool to microscopy methods on the one hand, but also to neutron scattering or hard X-ray resonant scattering, on the other 
hand. Moreover, recent applications have been developed to study the morphology of nano-scale devices such as organic junctions [77].

\section{Revealing new electronic properties}

On the experimental side, the starting point of the many developments in this field has been the observation of resonant scattering in $3 d$ and $4 f$ magnetic systems $[5,14,78]$, followed by the huge resonant effects found at $M$ edges of actinides [79]. Since then, REXS has become an ideal tool to analyse electronic properties of condensed matter, essentially because of its sensitivity to the electronic states (symmetry of ground and intermediate states, energy scales, ... ). The combination of these spectroscopic characteristics and the features of the elastic scattering methods has led to the burgeoning of exciting results.

Firstly, REXS has been exploited as an intensity enhancer in particular for magnetic structures determinations from powder diffraction experiments in the hard X-ray range $[80,81]$ and in the soft X-ray spectrum [82]. Whatever promising this technique is, the useful results have been so far restricted to few observed magnetic Bragg peaks which do not permit full ab-initio magnetic structure determinations. The strength of REXS is to complement neutron diffraction experiments with a much superior resolution in q-space and a wide variety of 'geometrical configurations' (polarisation effects, pure scattering geometry) that enables disentangling equivalent solutions for magnetic structures [83]. Selected resonant edges have moved from the 'easy' $7-20 \mathrm{keV}$ range that was exploited in the early days of REXS (with the notable exception of [14] where $\mathrm{K}$ edge resonant of $\mathrm{Ni}$ metal was used) to actinide range [79-84] but also to the soft X-ray range [85]. Furthermore, the REXS scattering amplitude contains terms that allow the uncovering of multi-q structures; clear signatures of couplings of Fourier components of the ordered magnetic moments with equivalent propagation vectors have been observed [84], leading to complete and unambiguous determinations of magnetic structures. It should be also noted that magnetic correlations at and near surfaces have been detected thanks to REXS methods [86-89].

Secondly, REXS can help disentangling different order parameters. This was clearly shown in the context of transition metal oxides where charges, lattice distortions, orbital occupancy and spins can exhibit long-range order [90-94]. The remarkable polarisation and energy dependences of the REXS scattering amplitude can even distinguish between order parameters that have similar symmetries. Until the development of REXS, there was no direct probe of the ordering of electronic orbitals, only secondary order parameters (lattice distortion effects or Jahn-Teller effects) could be readily detected with the use of standard scattering methods. A major impact of REXS has been to offer the possibility of experimental observations of orbital ordering and related phenomena [90-95]. In turn, these studies have led to deeper understanding of the resonant process, in particular at $\mathrm{K}$ edges of transition metal oxides $[96,97]$. Indeed, REXS has contributed greatly to the development of the electronic structure of materials with complex electronic properties where spontaneous magnetization and polarization coexist.

Thirdly, REXS has elucidated the origin of long-range order in some materials. High-order moment ordering has been identified in $5 f$ - [98] and $4 f$ - [99] systems. In $d$-electron materials, the existence of ordered anapole moments has been inferred from REXS data $[100,101]$.

Finally, the element specificity of REXS has been exploited to reveal contributions to magnetic scattering arising from 'non-magnetic' anions [84,102,103], although neutron scattering studies have indicated the absence of long-range ordered magnetic 
moments on these anions. These observations have provided pertinent information about the hybridization of electronic wavefunctions.

The energy dependence of REXS intensities [104] has not been yet fully exploited because of the complication related to the mixing of the real and imaginary parts of the scattering amplitudes, but also because of the moderate resolution of experiments and the complexity of the absorption corrections [105].

\section{Perspectives}

This succinct introduction to the REXS 2011 meeting in Aussois does not pretend to be exhaustive. It covers a selection of domains that are very much active today in the fields of materials sciences and electronic systems where many advances have made in the last decades. As predicted by D.H. Templeton at Malente [2], new topics have emerged and other applications of REXS will continue to materialise. The (partial) coherence of the X-ray beams is likely to play a major role also in REXS experiments. 'Coherent' REXS methods have been exploited at synchrotron sources [32] already, and also at FEL X-ray sources $[106,107]$. A few years before, it was noted [108] that the probed coherence volume could play an important role when it approached the size of macroscopically ordered regions in the sample. It can be anticipated that REXS combined with coherent X-ray beams will become an important feature in the field and will lead to exciting new results.

Although it is outside the scope of this meeting, Resonant Inelastic X-ray Scattering methods [11] should be mentioned as a very promising tool to probe magnetic excitations [109], an area that has been the realm of inelastic neutron scattering. In the field of dynamics, but on different time scales, the advent of X-ray lasers opens new avenues to time-resolved studies that exploit REXS methods to investigate magnetic systems [107].

Resonant scattering appears to be ubiquitous and it will continue to bring new exciting results, some of them will certainly arise from developments that took place during the last two or three decades and did not lead to visible advances; in particular, the observation of anomalous shift of diffraction peaks [110] in REXS experiments has not yet converted into new physics.

A final remark: the contributions to the REXS 2011 meeting in Aussois have amply demonstrated the remarkable achievements of REXS until today. Clearly, more is to be expected in the future!

\section{References}

1. Resonant Anomalous X-Ray Scattering, edited by G. Materlik, J. Spark, K. Fisher (Elsevier, Amsterdam, 1994)

2. D.H. Templeton, in Resonant Anomalous X-Ray Scattering, edited by G. Materlik, J. Spark, K. Fisher (Elsevier, Amsterdam, 1994), p. 1

3. R.W. James, The Optical Principles of the Diffraction of X-rays (Ox Bow Press, Woodbridge, 1962)

4. B. Lengeler, in Resonant Anomalous X-Ray Scattering, edited by G. Materlik, J. Spark, K. Fisher (Elsevier, Amsterdam, 1994), p. 35

5. D. Gibbs, D.R. Harshman, E.D. Isaacs, D.B. McWhan, D. Mills, C. Vettier, Phys. Rev. Lett. 61, 1241 (1988)

6. D.H. Templeton, L.K. Templeton, Acta Cryst. A 36, 237 (1980)

7. A. Kirfel, A. Petcov, K. Eichhorn, Acta Cryst. A 47, 180 (1991)

8. K.D. Finkelstein, Qun Shen, S. Shastri, Phys. Rev. Lett. 69, 1612 (1992) 
9. M. Blume, in Resonant Anomalous X-Ray Scattering, edited by G. Materlik, J. Spark, K. Fisher (Elsevier, Amsterdam, 1994), p. 495

10. P. Carra, B.T. Thole, Rev. Mod. Physics 66, 1509 (1994)

11. L.J.P. Ament, M. van Veenendaal, T.P. Deveraux, J.P. Hill, J. van den Brink, Rev. Mod. Physics 83, 705 (2011)

12. M. Guarise, B. Dalla Piazza, M. Morettei Sala, G. Ghiringhelli, L. Braicovich, H. Berger, J.N. Hancock, D. van der Marel, T. Schmitt, V.N. Strocov, L.J.P. Ament, J. van den Brink, P.H. Lin, P. Xu, H.M. Rønnow, M. Grioni, Phys. Rev. Lett. 105, 157006 (2010)

13. J. Goulon, A. Rogalev, F. Wilhelm, C. Goulon-Ginet, P. Carra, Phys. Rev. Lett. 88, $237401(2002)$

14. K. Namikawa, M. Ando, T. Nakajima, H. Kawata, J. Phys. Soc. Jpn. 54, 4099 (1985)

15. J.P. Hannon, G.T. Trammell, M. Blume, D. Gibbs, Phys. Rev. Lett. 61, 1245 (1988)

16. C. Brouder, P. Phys. Condens. Matter 2, 708 (1990)

17. S.W. Lovesey, E. Balcar, K.S. Knight, J. Fernández Rodríguez, Phys. Rep. 411, 233 (2005)

18. S. Di Matteo, Y. Joly, C.R. Natoli, Phys. Rev. B 72, 144406 (2005)

19. S.W. Lovesey, E. Balcar, J. Phys. Soc. Jpn. 79, 074707 (2010)

20. L. Paolasini, F. de Bergevin, C.R. Physique 9, 550 (2008)

21. J.P. Hill, D.F. McMorrow, Acta Cryst. A 52, 236 (1996)

22. C. Detlefs, C. Mazzoli (to be published)

23. Y. Joly, Eur. Phys. J. Special Topics 208, 21 (2012)

24. B.T. Thole, P. Carra, F. Sette, G. van der Laan, Phys. Rev. Lett. 68, 1943 (1992)

25. M. van Veenendaal, P. Carra, B.T. Thole, Phys. Rev. B 54, 16010 (1996)

26. D.H. Bilderback, P. Elleaume, H. Weckert, J. Phys. B: At. Mol. Opt. Phys. 38, S773 (2005)

27. J. Chavanne, P. Elleaume, in Proceedings of $10^{\text {th }}$ biannual European Particle Accelerator Conference (Edinburgh, 2006), p. 3290

28. A. Rogalev, J. Goulon, G. Benayoun, P. Elleaume, J. Chavanne, C. Penel, P. Van Vaerenbergh, Proc. SPIE 3773, 275 (1999)

29. C. Giles, C. Malgrange, J. Goulon, F. de Bergevin, C. Vettier, A. Fontaine, E. Dartyge, S. Pizzini, F. Baudelet, A. Freund, Rev. Sci. Instrum. 66, 1549 (1995)

30. C. Mazzoli, S.B. Wilkins, S. Bi Matteo, B. Detlefs, C. Detlefs, V. Scagnoli, L. Paolasini, P. Ghigna, Phys. Rev. B 76, 195118 (2007)

31. A. Snigerev, I. Snigereva, C.R. Physique 9, 507 (2008)

32. G. Beutier, A. Marty, F. Livet, A. Haznar, E. Dudzik, S. Stanescu, V. Chamard, G. van der Laan, New Journal Phys. 11, 113026 (2009)

33. J. Kokubun, M. Kanazawa, K. Ishida, V.E. Dmitrienko, Phys. Rev. B 78, 115112 (2008)

34. D.H. Templeton, L.K. Templeton, Phys. Rev. B 49, 14850 (1994)

35. V.E. Dmitrienko, E.N. Ovchinnnikova, J. Kokubun, K. Ishida, JETP Letters 92, 383 (2010)

36. Y. Tanaka, T. Takeuchi, S.W. Lovesey, K.S. Knight, A. Chainani, Y. Takata, M. Oura, Y. Senba, H. Ohashi, Y. Takata, Phys. Rev. Lett. 100, 145502 (2008)

37. L.S. Hirst, S.J. Watson, H.F. Gleeson, P. Cluzeau, P. Barois, R. Pindak, J. Pitney, A. Cady, P.M. Johnson, C.C. Huang, A.-M. Levelut, G. Srajer, J. Pollmann, W. Caliebe, A. Seed, M.R. Herbert, J.W. Goodby, M. Hird, Phys. Rev. E 65, 041705 (2002)

38. Hoyoung Jang, J.-S. Lee, K.-T. Ko, W.-S. Noh, T.Y. Koo, J.-Y. Kim, K.-B. Lee, J.-H. Park, C.L. Zang, Sung Bae Kim, S.-W. Cheong, Phys. Rev. Lett. 101, 047203 (2011)

39. J. Kokubun, M. Kanazawa, K. Ishida, V.E. Dmitrienko, Phys. Rev. B 64, 073203 (2001)

40. A. Kirfel, J. Grybos, V.E. Dmietrienko, Phys. Rev. B 66, 165202 (2002)

41. J. Goulon, N. Jaouen, A. Rogalev, F. Wilhelm, Ch. Goulon-Ginet, C. Brouder, Y. Joly, E.N. Ovchinnikova, V.E. Dmitrienko, J.Phys.: Condens. Matter 19, 156201 (2007)

42. A. Rogalev, J. Goulon, F. Wilhelm, C.R. Physique 9, 642 (2008)

43. B.T. Thole, P. Carra, F. Sette, G. van der Laan, Phys. Rev. Lett. 68, 1943 (1992)

44. P. Carra, R. Benoist, Phys. Rev. B 62, R7703 (2000)

45. J.M. Bijvoet, Proc. Acad. Sci. Amsterdam B52, 313 (1949) 
46. D.E. Cox, A.P. Wilkinson in Resonant Anomalous X-Ray Scattering, edited by G. Materlik, J. Spark, K. Fisher (Elsevier, Amsterdam, 1994), p. 195

47. W.A. Hendrickson, in Resonant Anomalous X-Ray Scattering, edited by G. Materlik, J. Spark, K. Fisher (Elsevier, Amsterdam, 1994), p. 159

48. J. Karle, in Resonant Anomalous X-Ray Scattering, edited by G. Materlik, J. Spark, K. Fisher (Elsevier, Amsterdam, 1994), p. 145

49. R. Kahn, Eur. Phys. J. Special Topics 208, 15 (2012)

50. W.A. Hendrickson, Science 254, 51 (1991)

51. R. Fourme, W. Sheppard, R. Kahn, Prog. Biophys. Molec. Biol. 64, 167 (1996)

52. J.R. Helliwell, J. Synchrotron Rad. 9, 1 (2000)

53. A.H. Moudden, D. Durand, M. Bessiere, S. Lefebvre, Phys. Rev B 37, 7655 (1988)

54. Y. Soejima, K. Yamasaki, K.F. Fischer, Acta Cryst. B 53, 415 (1997)

55. C. Vettier, D.B. McWhan, E.M. Gyorgy, J. Kwo, B.M. Buntschuh, B.W. Batterman, Phys. Rev. Lett. 56, 757 (1986)

56. M. Helliwell, J. Synchrotron Rad. 7, 139 (2000)

57. J.L. Hodeau, V. Favre-Nicolin, S. Bos, H. Rebevier, E. Lorenzo, J.F. Berar, Chem. Rev. 101, 1843 (2001)

58. V. Favre-Nicolin, S. Bos, J.E. Lorenzo, P. Bordet, W. Shepard, J.-L. Hodeau J. Appl. Cryst. 33, $52(2000)$

59. H. Stragier, J.O. Cross, J.J. Rehr, L.B. Sorensen Phys. Rev. Lett. 21, 3064 (1992)

60. I.J. Pickering, M. Sansone, J. March, G.N. George J. Am. Chem. Soc. 115, 6302 (1993)

61. H. Renevier, J.L. Hodeau, P. Wolfers, S. Andrieu, J. Weigelt, R. Frahm Phys. Rev. Lett. 78, 2775 (1997)

62. N.A. Katcho, M.I. Richard, O. Landré, G. Tourbot, M.G. Proietti, H. Renevier, V. Favre-Nicollin, B. Daudin, G. Chen, J.J. Zhang, B. Bauer, J. Phys.: Conference Series 190, 012129 (2009)

63. Gennesys White Paper, edited by H. Dosch, M.H. Van der Voorde (Max Plank Institute, Stuttgart, 2009), p. 399

64. R. Magalhães-Paniago, G. Medeiros-Ribeiro, A. Malachias, S. Kycia, T.I. Kamins, R. Stan Williams Phys. Rev. B 66, 245312 (2002)

65. T.U. Schülli, J. Stangl, Z. Zhong, R.T. Lechner, M. Sztucki, T.H. Metzger, G. Bauer, Phys. Rev. Lett. 90, 066105 (2003)

66. B. Lee, S. Seifert, S.J. Riley, G. Tikhonov, N.A. Tomczyk, S. Vajda, R.E. Winans, Jour. Chem. Phys. 123, 074701 (2005)

67. T. Vad, H.-G. Haubold, N. Waldhöfner, H. Bönnemann J. Appl. Cryst. 35, 459 (2002)

68. H. Palancher, J.-L. Hodeau, C. Pichon, J.F. Pichon, J.-F. Bérar, J. Lynch, B. Rebours, J. Rodriguez-Carvajal, Ang. Chemie 44, 1725 (20050)

69. S. Hosokawa, Y. Wang, W.-C. Pilgrim, J.-F. Bérar, S. Mamedov, P. Boolchand, J. NonCryst. Solids 352, 1517 (2006)

70. I. Pickering, M. Sansone, J. Marsch, G.N. George J.A.C.S. 115, 6302 (1993)

71. R. Kahn, Ph. Carpentier, C. Berthet-Colominas, M. Capitan, M.-L. Chesne, E. Fanchon, S. Lequien, D. Thiaudière, J. Vicat, P. Zielinski, H.B. Stuhrmann, J. Synchrotron Rad. 7, $131(2000)$

72. M. Sztucki, E. Di Cola, T. Narayanan, J. Appl. Cryst. 43, 1479 (2010)

73. H.B. Stuhrmann, Adv. Polym. Sci. 67, 123 (1985)

74. T. Araki, H. Ade, J.M. Stubbs, D.C. Sundberg, G.E. Mitchell, J.B. Kortright, A.L.D. Kilcoyne, Appl. Plys. Lett. 89, 124106 (2006)

75. C. Wang, T. Araki, H. Ade, Appl. Phys. Lett. 87, 214109 (2005)

76. H. Ade, Eur. Phys. J. Special Topics 208, 305 (2012)

77. S. Swaraj, C. Wang, H. Yan, B. Watts, J. Lüning, C.R. McNeill, H. Ade, Nano Lett. 10, $2863(2010)$

78. D. Gibbs, G. Grübel, D.R. Harshman, E.D. Isaacs, D.B. McWhan, D. Mills, C. Vettier, Phys. Rev. B 43, 5663 (1991)

79. D.B. McWhan, C. Vettier, E.D. Isaacs, G.E. Ice, D.P. Siddons, J.B. Hastings, C. Peters, O. Vogt, Phys. Rev. B 42, 6007 (1990) 
80. S.P. Collins, D. Laundy, C.C. Tang, R.J. Cernik, J. Phys.: Condens. Matter 7, L223 (1995)

81. J.W. Kim, L. Tan, D. Wermeille, S.L. Bud'ko, P.C. Canfield, A.I. Goldman, J. Phys.: Condens. Matter 17, L493 (2005)

82. U. Staub, M. Garciá-Fernández, A.M. Mulders, Y. Bodenthin, M.J. Martínez-Lope, J.A. Alonso, J. Phys.: Condens. Matter 19, 092201 (2007)

83. S. Langridge, J.A. Paixão, N. Bernhoeft, C. Vettier, G.H. Lander, D. Gibbs, S.Aa. Sørensen, A. Stunault, D. Wermeille, E. Talik, Phys. Rev. Lett. 82, 2187 (1999)

84. G.H. Lander, Eur. Phys. J. Special Topics 208, 129 (2012)

85. S.B. Wilkins, P.D. Hatton, M.D. Roper, D. Prabhakaran, A.T. Boothroyd, Phys. Rev. Lett. 90, 187201 (2003)

86. D. Gibbs, J.P. Hill, C. Vettier, Phys. Stat. Sol. 215, 667 (1999)

87. J.M. Tonnerre, L. Seve, D. Raoux, G. Soullie, B. Rodmacq, P. Wolfers, Phys. Rev. Lett. 75, 740 (1995)

88. G.M. Watson, D. Gibbs, G.H. Lander, B.D. Gaulin, L.E. Berman, Hj. Matzke, W. Ellis, Phys. Rev. Lett. 77, 751 (1996)

89. S. Ferrer, J. Alvarez, E. Lundgren, X. Torrelles, P. Fajardo, F. Boscherini, Phys. Rev. B 56, 9848 (1997)

90. Y. Murakami, J.P. Hill, D. Gibbs, M. Blume, I. Koyama, M. Tanaka, H. Kawata, T. Arima, Y. Tokura, K. Hirota, Y. Endoh, Phys. Rev. Lett. 81, 582 (1998)

91. J.E. Lorenzo, C. Mazzoli, N. Jaouen, C. Detlefs, D. Mannix, S. Grenier, Y. Joly, C. Marin, Phys. Rev. Lett. 101, 226401 (2008)

92. J. Blasco, J. García, G. Subías, Phys. Rev. B 83, 104105 (2011)

93. Y. Endoh, K. Hirota, S. Ishihara, S. Okamoto, Y. Murakami, A. Nishizawa, T. Fukuda, H. Kimura, H. Nojiri, K. Kaneko, S. Maekawa, Phys. Rev. Lett. 82, 4328 (1999)

94. K.J. Thomas, J.P. Hill, S. Grenier, Y.-J. Kim, P. Abbamonte, L. Venema, A. Rusydi, Y. Tomioka, Y. Tokura, D.F. McMorrow, G. Sawatky, M. van Veenendaal, Phys. Rev. Lett. 92, 237204, (2004)

95. I. Zegkinoglou, J. Strempfer, C.S. Nelson, J.P. Hill, J. Chakhalian, C. Bernhard, J.C. Lang, G. Strajer, H. Fukazawa, S. Nakatsuji, Y. Maeno, B. Keimer, Phys. Rev. Lett. 95, $136401(2005)$

96. I.S. Elfimov, V.I. Anisimov, G.A. Sawatsky, Phys. Rev. Lett. 82, 4264 (1999)

97. M. Benfatto, Y. Joly, C.R. Natoli, Phys. Rev. Lett. 83, 636 (1999)

98. S.B. Wilkins, R. Caciuffo, C. Detlefs, J. Rebizant, E. Colineau, F. Wastin, G.H. Lander, Phys. Rev. B 73, 060406 (2006)

99. O. Bunau, R.-M. Galéra, Y. Joly, M. Amara, S.E. Luca, Phys. Rev. B 81, 144402 (2010)

100. J. Fernández-Rodríguez, V. Scagnoli, C. Mazzoli, F. Fabrizi, S.W. Lovesey, J.A. Blanco, D.S. Sivia, K.S. Knight, F. de Bergevin, L. Paolasini, Phys. Rev. B 81, 085107 (2010)

101. V. Scagnoli, U. Staub, Y. Bodenthin, R.A. de Souza, M. García-Fernández, M. Garganourakis, A.T. Boothroyd, D. Prabhakaran, S.W. Lovesey, Science 332, 696 (2011)

102. D. Mannix, A. Stunault, N. Bernhoeft, L. Paolasini, G.H. Lander, C. Vettier, F. de Bergevin, D. Kaczorowski, A. Czopnik, Phys. Rev. Lett. 86, 4128 (2001)

103. K. Kuzushita, K. Ishii, S.B. Wilkins, B. Janousova, T. Inami, K. Ohwada, M. Tsubota, Y. Murakami, K. Kaneko, S. Ikeda, Y. Haga, Y. Onuki, N. Bernhoeft, G.H. Lander, Phys. Rev. B 73, 104431 (2006)

104. J.-I. Igarashi, M. Takahshi, J. Phys. Soc. Jpn. 69, 4087 (2000)

105. A. Stunault, F. de Bergevin, D. Wermeille, C. Vettier, Th. Brückel, N. Bernhoeft, G.J. McIntyre, J.Y. Henry. Phys. Rev. B 60, 10170 (1999)

106. C. Gutt, S. Streit-Nierobisch, L.-M. Stadler, B. Pfau, C.M. Günther, R.Könnecke, R. Frömter, A. Kobs, D. Stickler, H.P. Oepen, R.R. Fäustlin, R. Treusch, J. Feldhaus, E. Weckert, I.A. Vartanyants, M. Grunze, A. Rosenhahn, T. Wilhein, S. Eisebitt, G. Grübel, Phys. Rev. B 81, 100401 (2010)

107. M. Altarelli, Eur. Phys. J. Special Topics 208, 351 (2012) 
108. N. Bernhoeft, A. Hiess, S. Langridge, A. Stunault, D. Wermeille, C. Vettier, G.H. Lander, M. Huth, M. Jourdan, H. Adrian, Phys. Rev. Lett. 81, 3419 (1998)

109. L. Braicovich, J. van den Brink, V. Bisogni, M. Moretti Sala, L.J.P. Ament, N.B. Brookes, G.M. De Luca, M. Salluzzo, T. Schmitt, V.N. Strocov, G. Ghiringhelli, Phys. Rev. Lett. 104, 077002 (2010)

110. N. Bernhoeft, G.H. Lander, M.J. Longfield, S. Langridge, D. Mannix, S.D. Brown, W.J. Nuttall, A. Hiess, C. Vettier, P Lejay, J. Phys.: Condens. Matter 16, 3869 (2004) 